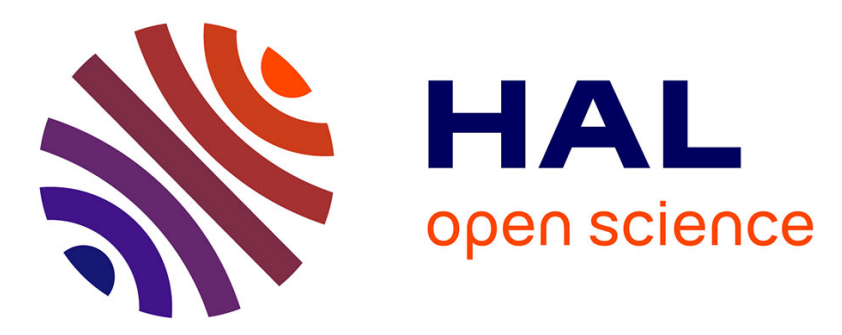

\title{
Détermination des profils acoustiques d'un fond marin de faible profondeur à partir du champ observé dans le chenal en présence d'une source donnée
}

\author{
M. Lambert, D. Lesselier
}

\section{- To cite this version:}

M. Lambert, D. Lesselier. Détermination des profils acoustiques d'un fond marin de faible profondeur à partir du champ observé dans le chenal en présence d'une source donnée. Journal de Physique IV Proceedings, 1994, 04 (C5), pp.C5-1067-C5-1070. 10.1051/jp4:19945234 . jpa-00252921

\author{
HAL Id: jpa-00252921 \\ https://hal.science/jpa-00252921
}

Submitted on 1 Jan 1994

HAL is a multi-disciplinary open access archive for the deposit and dissemination of scientific research documents, whether they are published or not. The documents may come from teaching and research institutions in France or abroad, or from public or private research centers.
L'archive ouverte pluridisciplinaire HAL, est destinée au dépôt et à la diffusion de documents scientifiques de niveau recherche, publiés ou non, émanant des établissements d'enseignement et de recherche français ou étrangers, des laboratoires publics ou privés. 


\title{
Détermination des profils acoustiques d'un fond marin de faible profondeur à partir du champ observé dans le chenal en présence d'une source donnée
}

\author{
M. LAMBERT et D. LESSELIER
}

Division Ondes, Laboratoire des Signaux et Systèmes, CNRS-ESE, 91192 Gif-sur-Yvette cedex, France

\begin{abstract}
Résumé : La caractérisation acoustique d'un fond marin dans une situation de petits fonds est considérée. La modélisation du fond est la suivante : une couche fluide homogène (l'eau) reposant sur une couche fluide inhomogène (sédiment) suivie d'un demi-espace fluide homogène (le socle). Ses paramètres acoustiques (vitesse des ondes de compression, densité) ne dépendent que de la profondeur. Une source cylindrique et une ligne horizontale de récepteurs sont placées dans l'eau. Les données sont les champs de pression en amplitude et en phase sur les hydrophones à plusieurs fréquences (de quelques $\mathrm{Hz}$ au $\mathrm{kHz}$ ). La fonction de Green du milieu stratifié peut être obtenue par transformée de Fourier du champ sur l'ouverture synthétique que constitue le réseau. A partir de ses valeurs aux faibles nombres d'ondes, le coefficient de réflexion en ondes planes du fond se construit à plusieurs fréquences et incidences précritiques. Ce coefficient est alors utilisé dans les processus d'inversion permettant de remonter aux paramètres du fond.
\end{abstract}

Abstract: The acoustical characterization of the seabed in shallow water is aimed at. The seabed is modeled as follows: a homogeneous fluid layer (water) lying on a inhomogeneous fluid layer (sediment) above a homogeneous fluid half-space (subbottom). Acoustical parameters (compressional wave velocity, density) depend on the depth only. A cylindrical source and horizontal ligne of receivers are placed in the water. The data are the pressure field amplitude and phase on the hydrophone at several frequencies (from a few $\mathrm{Hz}$ to one $\mathrm{kHz}$ ). The Green function of the stratified medium can be obtained by Fourier transform of the field. From the result at low wavenumbers, the plane wave reflection coefficient of the seabed is reconstructed at several frequencies and precritical angles. It can then be used as data in inversion algorithms retrieving the seabed parameters.

\section{INTRODUCTION}

Ce travail porte sur l'obtention du coefficient de réflexion en ondes planes d'un fond marin par petits fonds à partir du champ de pression recueilli sur une ligne horizontale d'hydrophones et son utilisation comme donnée permettant de remonter à ses paramètres acoustiques via des processus développés dans notre laboratoire [1]. Rappelons en effet que ces paramètres jouent un rôle prépondérant dans la propagation des ondes sonores lorsqu'il est à faible profondeur [2] et que les difficultés de mesure à partir d'échantillons prélevés sont réelles. D'où l'intérêt à priori d'un tel diagnostic, cela même quand le problème de référence est, comme ici, la très étudiée reconstruction de milieux 1-D.

\section{MODÉLISATION}

Le modèle est précisé figure 1. Le fond est assimilé à une couche horizontale inhomogène (un sédiment) reposant sur un socle homogène semi-infini. L'eau est modélisée par une couche horizontale homogène d'épaisseur finie $h$, l'interface air/eau étant parfaitement réfléchissante. Les milieux sont des fluides linéaires et isotropes de densités $\rho$. Seules des ondes de compression sont supportées, de célérités $c$. Par construction $c$ et $\rho$ ne dépendent que de la profondeur $z$ et sont constantes au delà d'un certain $d$ qui, de fait, détermine la position de l'interface sédiment/socle. Les pertes, s'il y a, varient linéairement avec la fréquence et interviennent dans la constante de propagation $k=\omega / c+j \omega \alpha$ (dépendance en $\exp (-j \omega t)$ ). Une source 
cylindrique et une ligne horizontale de récepteurs sont placées respectivement aux points $\left(z_{s}, 0\right)$ et $(z, x)$.

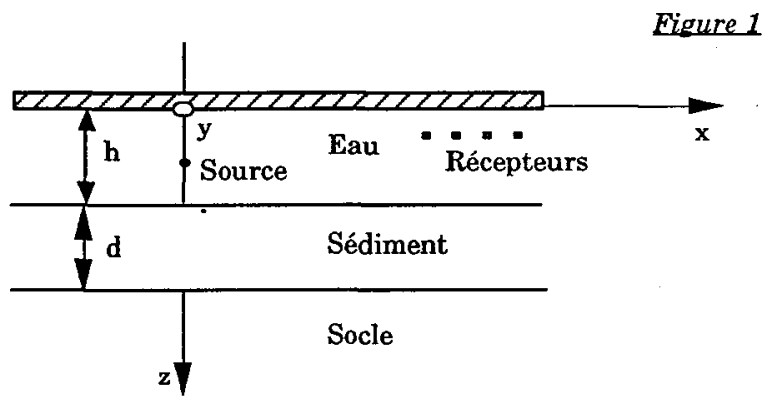

\section{METHODE DE CALCUL DE $\dot{p}(x, z, \omega), g\left(z, z_{3}, k_{x}\right), R_{f}\left(k_{x}\right)$ et $R \theta$}

Le champ de pression, $p(x, z, \omega)$, dans l'eau en un point $(z, x)$ est la transformée de Fourier inverse (1) de la fonction de Green du milieu (2), $g\left(z, z_{s}, k_{x}\right)$ :

$$
\begin{gathered}
p(x, z, \omega)=\frac{1}{2 \pi} \int_{-\infty}^{+\infty} g\left(z, z_{s}, k_{x}\right) \exp \left(j k_{x} x\right) d k_{x} \\
g\left(z, z_{s}, k_{x}\right)=-\frac{1}{2 j \beta_{0}}\left[\exp \left(j \beta_{0}\left|z-z_{s}\right|\right)-\exp \left(j \beta_{0}\left(z+z_{s}\right)\right)\right]+\frac{2 \sin \left(\beta_{0} z_{s}\right) \sin \left(\beta_{0} z\right) R_{f}\left(k_{x}\right) \exp \left(2 j \beta_{0} h\right)}{j \beta_{0}\left[1+R_{f}\left(k_{x}\right) \exp \left(2 j \beta_{0} h\right)\right]}
\end{gathered}
$$

où $k_{x}$ est le nombre d'onde horizontal dépendant de la fréquence circulaire w et $\beta_{0}$ le nombre d'onde vertical dans l'eau de paramètres $c_{0}$ et $\rho_{0}$ et de constante de propagation $k_{0}=\sqrt{ } \beta_{0}^{2}+k_{x}^{2}\left(\lambda_{0}=2 \pi / k_{0}\right)$, et $R_{f}\left(k_{x}\right)$ le coefficient de réflexion en ondes planes du milieu stratifié constitué par l'empilement sédiment/socle à fréquence $f$ fixe et $k_{x}$ variable.

Nombre de théories et codes ont été développés pour le calcul du champ de pression dans la lame d'eau [3]. Nous nous sommes particulièrement intéressés à la théorie des modes qui est très adaptée au cas de la propagation des ondes sonores par petits fonds [4]. Le contour d'intégration de (1) est déplacé dans le plan complexe. La transformée de Fourier devient une somme finie (grâce au choix de la coupure EJP) de modes solutions de l'équation caractéristique donnée par le dénominateur du deuxième terme de (2) et d'intégrales de branches liées aux vecteurs de propagation du milieu semi-infini (le socle). La prise en compte des intégrales de branche est capitale pour une bonne connaissance de $p(x, z, \omega)$ près de la source, très important dans notre cas. Les pôles du dénominateur (modes propagatifs) sont recherchés grâce à un algorithme fourni par A. Tijhuis et basé sur le théorème de Cauchy.

$p(x, z, \omega)$ connu sur un certain domaine d'espace dont le point de départ est assez proche de la source, il est. possible de calculer $g\left(z, z_{s}, k_{x}\right)$ en inversant (1), grâce à l'application d'une TFR utilisant un filtre de Hamming. Après avoir soustrait le premier terme du membre de droite, nous nous retrouvons alors avec la partie de la fonction de Green appelée $g_{f o n d}\left(z, z_{s}, k_{x}\right)$ faisant intervenir $R_{f}\left(k_{x}\right)$ (équivalent à $R_{f}(\theta)$, déduit de $k_{x}=k_{0} \sin \theta$ pour les $k_{x}$ réels, $k_{0}$ dépendant de $f$ ), seul élément lié au fond marin. D'où

$$
R_{f}\left(k_{x}\right)=\frac{2 \sin \left(\beta_{0} z_{s}\right) \sin \left(\beta_{0} z\right)-j \beta_{0} g_{\text {fond }}\left(z, z_{s}, k_{x}\right)}{j \beta_{0} g_{\text {fond }}\left(z, z_{s}, k_{x}\right)} \exp \left(2 j \beta_{0} h\right)
$$

calcul qui ne pose aucun problème excepté lorsque $g_{\text {fond }}\left(z, z_{s}, k_{x}\right)$ est nul, ce qui survient lorsque

$$
f_{c}^{(k)}=\frac{k c_{0}}{2 z \cos (\theta)}
$$

où $\kappa$ est un entier et $z$ peut être remplacé par $z_{s}$ (les deux jouant un rôle symétrique).

Cette opération répétée pour $N$ fréquences mène à autant de coefficients de réflexion $R(\theta)$; le coefficient de réflexion en ondes planes $R_{\theta}(f)$ à fréquence $f$ variable et angle d'incidence $\theta$ fixe à ces $N$ fréquences en est alors déduit. C'est à partir de la connaissance de ce coefficient à des angles pré-critiques que nous avons 
développé des processus de reconstruction "Born-Itératif" ou "Born-Etendu" [2].

\section{QUELQUES RAPPELS SUR LES PROCESSUS D'TNVERSION BORN-ITERATIF ET BORN-ETENDU}

Ils s'appuient sur le changement de variable profondeur réelle $z \rightarrow$ profondeur apparente $s$ [5], faisant que l'inconnue à déterminer n'est alors plus $c(z)$ et $\rho(z)$ mais un indice $N_{\theta}(s)$ :

$$
s(z)=\int_{0}^{z} \frac{\rho\left(z^{\prime}\right)}{\rho_{0}} d z^{\prime} \text { et } N_{\theta}(s)=\frac{\rho_{0}}{\rho(s)} \sqrt{\frac{c_{0}^{2}}{c^{2}(s)}-\sin ^{2} \theta}
$$

la connaissance des indices à deux angles différents permettant de remonter aux paramètres acoustiques. Les deux processus utilisés pour remonter à ces indices ont été développés à partir de l'approximation de Born sans s'y réduire : le premier, Born-Itératif, est une méthode itérative de résolution du problème linéarisé, un terme de régularisation permettant de sélectionner la solution la plus "lisse" parmi toute les solutions possibles alors que le second, Born-Etendu, est une méthode directe mettant en jeu une relation de Fourier entre le coefficient de réflexion en ondes planes et un contraste, fonction de l'indice $N_{\theta}(s)$, à laquelle s'ajoutent des corrections de niveau et d'élongation augmentant la gamme des contrastes à reconstruire.

\section{RESULTATS NUMÉRIQUES}

La modélisation choisie est la suivante: une source de profondeur $z_{s}=6 \mathrm{~m}$ rayonne à la fréquence $103 \mathrm{~Hz}$, une série de 512 récepteurs est placée à une profondeur $z=7 \mathrm{~m}$ sur une distance de 0 à $1000 \mathrm{~m}$, la couche d'eau est d'épaisseur $h=30 \mathrm{~m}$, de densité $\rho_{0}=1000 \mathrm{~kg} / \mathrm{m}^{3}$ et de célérité $c_{0}=1500 \mathrm{~m} / \mathrm{s}$, le sédiment et le socle sont de même densité $\rho=1500 \mathrm{~kg} / \mathrm{m}^{3}$, célérité $c=2000 \mathrm{~m} / \mathrm{s}$ et atténuation $\alpha=0$.

Nous observons une bonne reconstruction dans l'ensemble de $g_{f o n d}\left(z, z_{s}, k_{x}\right)$ (Fig. 2b) avec une réserve pour la description des pics expliquée par l'utilisation du champ proche (Fig. 2a) et non du champ lointain. Le coefficient de réflexion $R_{f}(\theta)$ obtenu (Fig. 2c) est satisfaisant pour les petits angles d'incidences, les oscillations sont dues au phénomène de Gibbs apparaissant dans la transformation de Fourier entre $p(x, z, \omega)$ et $g\left(z, z_{s}, k_{x}\right)$ et ce malgré l'utilisation du filtre de Hamming. L'opération répétée 20 fois, le coefficient de réflexion $R_{\theta}(f)$ à $0^{\circ}$ et $40^{\circ}$ est obtenu (Fig. 3a) (la valeur du coefficient à $125 \mathrm{~Hz}$ et $0^{\circ}$ satisfait (4) et ne peut donc pas être pris en compte dans la suite du processus).

C'est à partir de $R_{\theta}(f)$ que les processus d'inversion ont été développés avec une étape supplémentaire pour Born-Etendu qui nécessite sa connaissance à un plus grand nombre de fréquences (256), ici par interpolation à partir des 20 calculées. Densité et célérité construites à partir de ces données, sans bruit, sont relativement satisfaisantes (Fig. $3 \mathrm{~b}$ ).

Nous nous sommes intéressés au devenir d'un tel processus en présence du bruit et pour cela nous avons envisagé un positionnement aléatoire des récepteurs (sujet aux mouvements de la mer) dans un rectangle de $1 \mathrm{~m}$ de côté. Si ni $p(x, z, \omega)$, ni $g_{\text {fond }}\left(z, z_{s}, k_{x}\right)$ (en particulier la position des pics) ne semblent modifiés par ce bruit, le coefficient $R_{f}(\theta)$ lui est franchement bruité et semble inutilisable (Fig. 4).

En conclusion, le procédé présenté est trop sensible au bruit tel quel et nécessite des améliorations. Il est cepedant loin d'être inintéressant, e.g., [5], à partir du champ de pression, obtient un coefficient de réflexion relativement correct. L'utilisation de la fonction de Green dans des processus d'inversion est, elle, intéressante grâce à la stabilité de la position des pics vis-à-vis du bruit, pics qui caractérisent le milieu.

\section{Références}

[1] LAMBERT, M. et al., 2ème Congrès SFA, Suppl. J. Phys. III, 2 (1992) 945-948.

[2] BREKHOVSKIKH, L. M. et LYSANOV, Y., Fundamentals of Ocean Acoustics (Springer, 1982).

[3] BUCKINGHAM M. J., J. Acoust. 5 (1992) 223-287.

[4] PORTER M., Saclantcen Memorandum SM-245 (1991).

[5] GODIN, O. A., Wave Motion 7 (1985) 515-528.

[6] GLATTETRE J. et al., J. Acoust. Soc. Am. 86 (1989) 680-690. 


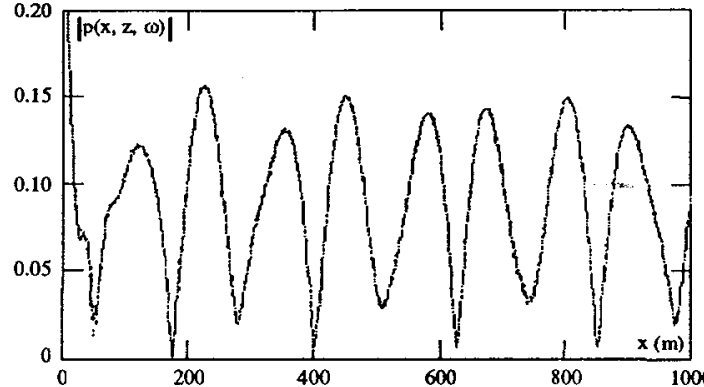

(a)

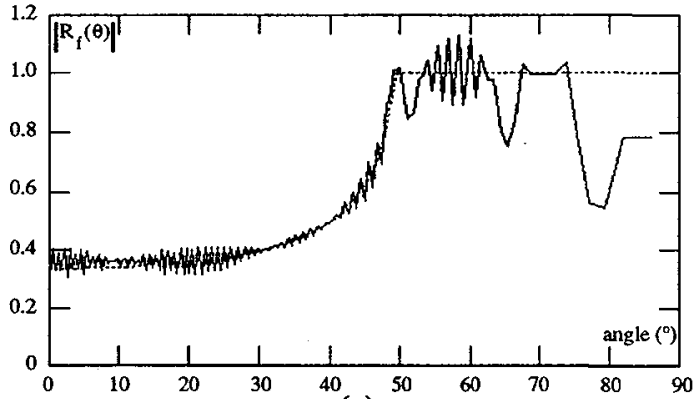

(c)

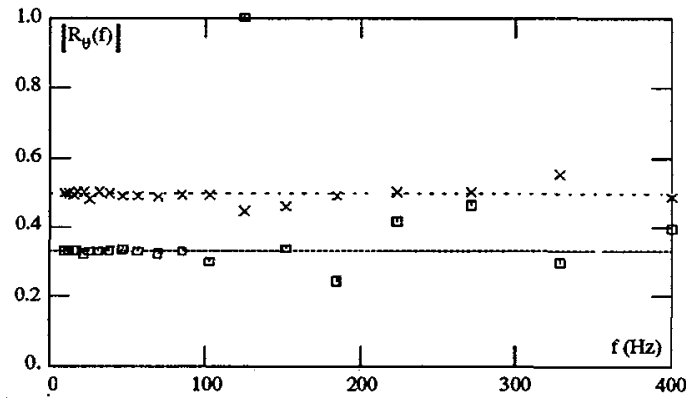

(a)

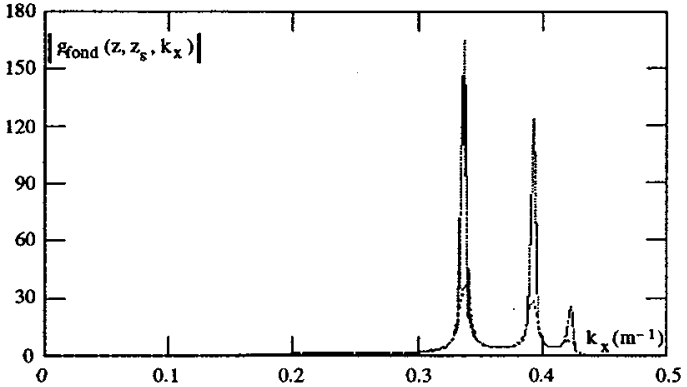

(b)

Fig.2 : processus d'obtention du coefficient de réflexion en ondes planes $R_{f}(\theta)$ à partir du champ de pression $p(x, z, \omega)$.

(a) module du champ de pression $p(x, z, \omega)$ à $103 \mathrm{~Hz}$ de 0 à $1000 \mathrm{~m}$ calculé par la méthode des modes.

(b) module de la fonction de Green $g_{\text {fond }}\left(z, z_{s}, k_{x}\right)$ exact $(-)$ et reconstruit (...) obtenu à partir de $p(x, z, \omega)$ de (a).

(c) coefficient de réflexion $R_{f}(\theta)$ exact (....) et reconstruit (-).

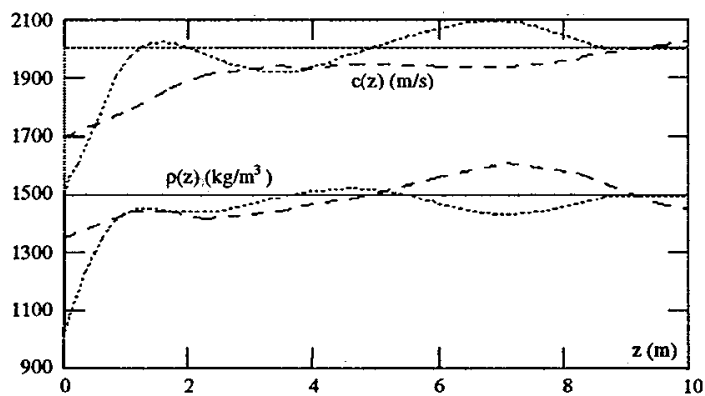

(b)

Fig.3 : Processus d'inversion à partir du coefficient de réflexion en ondes planes en fonction de l'angle d'incidence $\theta, R_{\theta}(f)$.

(a) module de $R_{\theta}(f)$ à $0^{\circ}$ (exact (-) et reconstruit (carré)) et à $40^{\circ}$ (exact (...) et reconstruit (croix)) pour 20 fréquences obtenu en répétant 20 fois le processus présenté figure 2.

(b) Densité et célérité exactes (-) et reconstruites par Born-Itératif (....) et Born-Etendu (-.--) à partir de $R_{\theta}(f)$ à $0^{\circ}$ et $40^{\circ}$.

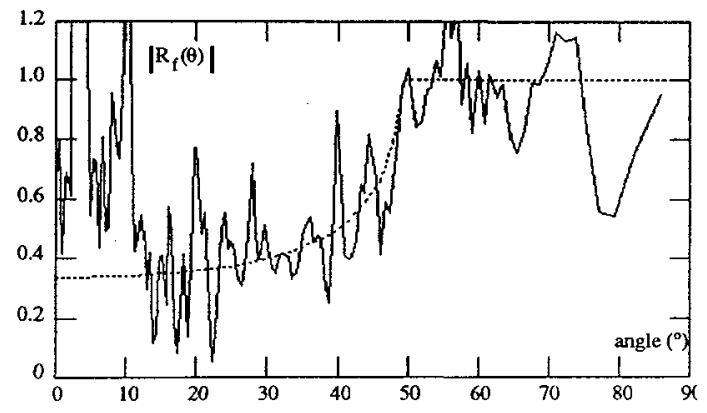

Fig.4 : module du coefficient de réflexion $R_{f}(\theta)$ exact (....) et reconstruit ( - ) à partir d'un champ de pression $p(x, z, \omega)$ bruité à $103 \mathrm{~Hz}$. Le bruit est dû à une méconnaissance de la position des récepteurs. 